\title{
Reduction of the Coherent Betatron Oscillation Amplitude by an RF Electric Field for the Fermilab Muon $g-2$ Experiment
}

\author{
On Kim ${ }^{* 1,2}$, Jihoon $\mathrm{Choi}^{1}$, Selcuk Haciomeroglu ${ }^{1}$, SeungPyo Chang ${ }^{1,2}$, Soohyung \\ Lee $^{1}$, Young-Im Kim ${ }^{1}$, Yannis K. Semertzidis ${ }^{1,2}$ \\ 1. Center for Axion and Precision Physics Research, IBS, Daejeon 34051, Republic of Korea \\ 2. Department of Physics, KAIST, Daejeon 34141, Republic of Korea \\ E-mail: bigstaron@kaist.ac.kr, pury828@gmail.com, \\ selcuk.haciomeroglu@gmail.com, koreanbrlekaist.ac.kr, \\ soohyungleedibs.re.kr, youngimeibs.re.kr, yannis@kaist.ac.kr
}

\section{Martin Gaisser}

Department of Physics, RWTH Aachen University, Aachen 52062, Germany

E-mail: gaisser@physik.rwth-aachen.de

\section{Yuri F. Orlov}

Department of Physics, Cornell University, New York 14853-2501, USA

E-mail: yuri.orlov@cornell.edu

\begin{abstract}
The Fermilab Muon g-2 experiment aims to measure the muon anomalous magnetic moment with a 140 parts-per-billion precision to investigate the greater than 3 standard deviation difference between the Standard Model prediction and the previous measurement by the BNL Muon g2 experiment. The coherent betatron oscillation (CBO) beam effects must be corrected for in the decay positron time spectra fits used in high precision muon storage ring based anomalous magnetic moment measurements. This MC simulation study indicates that the application of radio frequency (RF) electric fields to the muon storage ring beam can reduced the CBO amplitude by up to a factor of 10, as well to increase the symmetry of the beam phase space. This is achieved by correcting the mismatched oscillation phases between the high and low momentum muon populations by modulating the muon beam betatron oscillation frequencies with off-resonance RF fields.
\end{abstract}

ICHEP 2018, International Conference on High Energy Physics

4-11 July 2018

Seoul, Republic of Korea

\footnotetext{
*Speaker.
} 


\section{Beam Phase Space Modulation with an RF Electric Field}

The muons with energy $3.09 \mathrm{GeV}$ are generated and stored in a storage ring in the Fermilab muon $g-2$ experiment. Until they decay into the electrons after $64.4 \mu$ s of the lifetime and the detector captures the electrons to measure the spin precession frequency of the muons, they oscillate both in a horizontal and a vertical direction. While the detector acceptance is significantly affected by the horizontal offset from the detector to the muon when it decays, one of the biggest systematic errors comes from the amplitude of the muon horizontal oscillation relative to the detector, which is called as Coherent Betatron Oscillation (CBO). This MC simulation study revealed that the CBO amplitude can be reduced by a factor of 10 by applying the RF electric field with $\mathrm{CBO}$ frequency $(\sim 420 \mathrm{kHz})$.

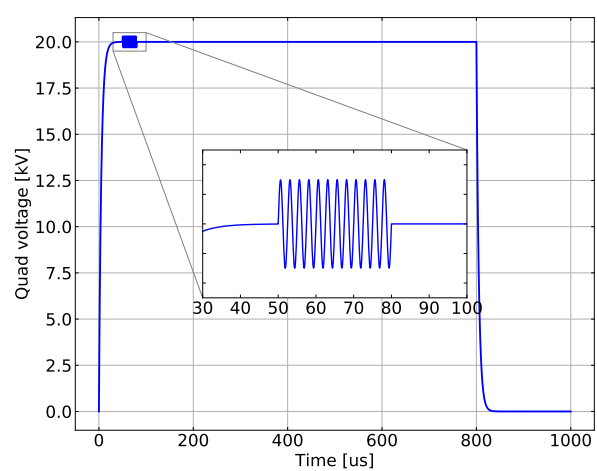

(a) High voltages on the electrostatic quadrupoles inside the $g-2$ storage ring during the storage time. Small modulation of the high voltage pulser with RF electric field of $\mathrm{CBO}$ frequency is applied at the early stage of the storage period.

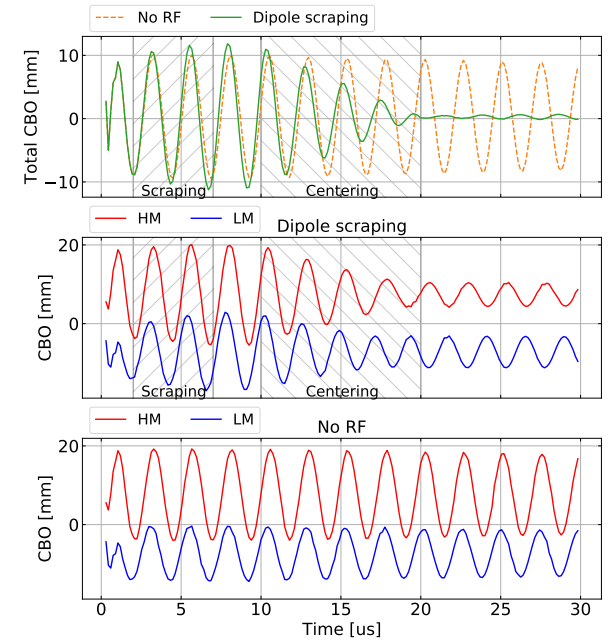

(b) From bottom to top: CBO for high momentum (HM, $\delta p / p>0)$ and low momentum (HM, $\delta p / p>0)$

muons without RF field; with RF field; total CBO for all muons with RF field.

Figure 1: Expected period and simulated effect of the RF electric field.

Figure 1 (a) illustrates how the RF signal will modulate the original static electric quadrupole fields for several tens of microseconds. Figure 1 (b) is the simulation result of 30,000 muons comparing the CBO amplitude between the cases when the RF field is or is not applied. The grey shaded regions are when the RF field is applied, and it shows how it becomes resonant to the muon transverse motion and match the $\mathrm{CBO}$ amplitude the same and phase oppsite for the high and low momentum muons to reduce the CBO amplitude by a factor of 10 .

\section{Acknowledgement}

This work was supported by IBS-R017-D1-2018-a00 of the Republic of Korea.

We thank our E989 collaborators, and Fermi National Accelerator Laboratory (Fermilab), a U.S. Department of Energy, Office of Science, HEP User Facility for the resources provided. 